Gut, 1981, 22, 217-222

\title{
Anomalies of peristalsis in idiopathic diffuse oesophageal spasm
}

\author{
M D KAYE \\ From the University of Vermont College of Medicine, Burlington, Vermont, USA
}

SUMMARY Oesophageal manometry revealed 'interrupted peristalsis'-proximal sequential contraction with simultaneous pressure increases in the mid-oesophagus and sequential contraction distally - and abnormally slow distal propagation of peristalsis in seven of a consecutive series of 12 patients with idiopathic diffuse oesophageal spasm (IDOS). These two abnormalities occurred together in six patients. It is proposed that 'interruption' of peristalsis and 'slow' distal propagation are apparent rather than real, and that they reflect failure of normal luminal obliteration during oesophageal contraction. This argument is extended to the 'aperistalsis' of achalasia. It is also proposed that IDOS, achalasia, and related idiopathic motor disorders may share a common pathogenetic abnormality-namely, functional obstruction at the level of the lower oesophageal sphincter. According to this hypothesis, the presentation of the patient and the progression of his disease are determined by the degree and duration of that obstruction, and the functional reserve of the oesophageal body musculature.

Idiopathic diffuse oesophageal spasm is characterised by symptoms of chest pain, dysphagia, or both and by radiographic abnormalities ranging from tertiary waves to the classical 'corkscrew' oesophagus. Manometric abnormalities are variable; and there are no uniform, generally accepted criteria for manometric diagnosis. Most would agree, however, that the typical patient with IDOS has powerful, prolonged, repetitive, and simultaneous contractions in the lower half or two-thirds of the oesophageal body. ${ }^{1}$ Some, ${ }^{2}$ but not all, would insist that the absence of peristalsis - that is, axially sequential contractionin this segment excludes IDOS. In such patients, the distinction between IDOS and 'vigorous achalasia' becomes blurred. Another apparent variant of IDOS, initially described by Pope $^{4}$ and subsequently given, by Benjamin et al., 5 the appellation 'nutcracker oesophagus', is characterised by powerful, prolonged but uniformly sequential distal oesophageal contractions.

A review of manometric records obtained at the University of Vermont from patients with IDOS during the 18 month period from January 1979 to

Address for correspondence: Dr Michael D Kaye, Given Building, C307, University of Vermont, College of Medicine, Burlington, VT 05405, USA.

Received for publication 29 October 1980
June 1980 revealed two additional interesting abnormalities. One, the occurrence of simultaneous pressure waves in the mid-oesophagus with sequential contraction below that level, hereafter referred to as 'interrupted peristalsis', has not to my knowledge been described before. The other, abnormally slow propagation of oesophageal contraction in the distal segment, was reported previously by us in certain patients with Parkinson's disease. ${ }^{6}$ A description of these abnormalities and discussion of their interpretation form the basis of this report.

\section{Methods}

PATIENTS WITH IDOS

Between January 1979 and June 1980, a diagnosis of idiopathic diffuse oesophageal spasm (IDOS), initially suspected on the basis of clinical and radiographic findings, was established by manometry in 12 patients. This group comprised nine women and three men. Ages ranged from 29 to 89 years, with a mean of 61.6 years. One patient presented with odynophagia alone. Each of the remaining 11 complained of dysphagia, which was associated with chest pain in five, eructation in two, regurgitation of food in one, and weight loss in one. None had symptoms of gastroesophageal reflux. In the one 
patient whose barium swallow was reported to be normal, manometry revealed extremely powerful (up to $470 \mathrm{~mm} \mathrm{Hg} ; 62.5 \mathrm{kPa}$ ), repetitive pressure waves, which were frequently simultaneous, in the lower half of the oesophageal body. Radiographic evidence of incoordination, ranging from prominent tertiary waves to a classical 'corkscrew' oesophagus, was present in the remaining 11 . In addition, five had an obvious hiatal hernia, two a B (Schatzki's) ring, two had an A ring, and one had a lower oesophageal diverticulum. Manometry in these patients revealed powerful simultaneous pressure waves, which were frequently prolonged, repetitive, or both, in the distal half to two-thirds of the oesophageal body. Intermittent sequential contractions were recorded from the distal oesophagus in 10 of the 12 patients. All showed sequential contraction in the proximal oesophagus.

\section{COMPARISON GROUP}

In order to provide a frame of reference for the interpretation of measurements in patients with IDOS, data on the manometric characteristics of lower oesophageal contraction in 10 healthy subjects are presented. This group comprised nine men and one woman. Ages ranged from 19 to 38 years. None had oesophageal symptoms. All gave informed consent, and performance of manometric studies in them was approved by the University of Vermont Committee on Human Experimentation for the Medical Sciences.

\section{OESOPHAGEAL MANOMETRY}

Patients with IDOS were studied with a commercially manufactured tube assembly (Arndorfer Medical Specialties, Greenland, Wisconsin). This has four openings for pressure transmission at the same axial level of the assembly, with four additional openings spaced at $5 \mathrm{~cm}$ intervals above that point. Normal subjects were studied with a simple triple-lumen tube, with openings spaced at $5 \mathrm{~cm}$ intervals. Each lumen was perfused at a rate of $0.6 \mathrm{ml} / \mathrm{min}$ by a pneumohydraulic capillary infusion system (Arndorfer Medical Specialties). Pressures were measured by external transducers (Bell and Howell P 327-1), the outputs of which were recorded on a Gilson Macropolygraph multichannel recorder. Respiration and swallowing were monitored by pneumograph and myograph respectively.

In patients with IDOS, lower oesophageal sphincter pressures (LOSP) were measured simultaneously by the four sensors located at the same axial level of the assembly. A station withdrawal method was used. LOS pressures were expressed as the mean of the end-expiratory measurements obtained by the four sensors, with gastric baseline pressure being used as zero reference. Oesophageal contractile responses to 'dry' and 'wet' $(5 \mathrm{ml}$ boluses of water at room temperature) swallows were then recorded by openings spaced at $5 \mathrm{~cm}$ intervals.

In normal subjects, the responses to 10 'dry' and 10 'wet' swallows were recorded with pressure sensors 2,7 , and $12 \mathrm{~cm}$ above the LOS.

\section{Results}

Lower oesophageal sphincter pressures in the group of patients with IDOS tended to be high. They ranged from 2.0 to $7.58 \mathrm{kPa}(15$ to $57 \mathrm{~mm} \mathrm{Hg}$ ) with a mean of $3.57 \mathrm{kPa}(31.5 \mathrm{~mm} \mathrm{Hg})$. It should be emphasised that these are end-expiratory pressures,

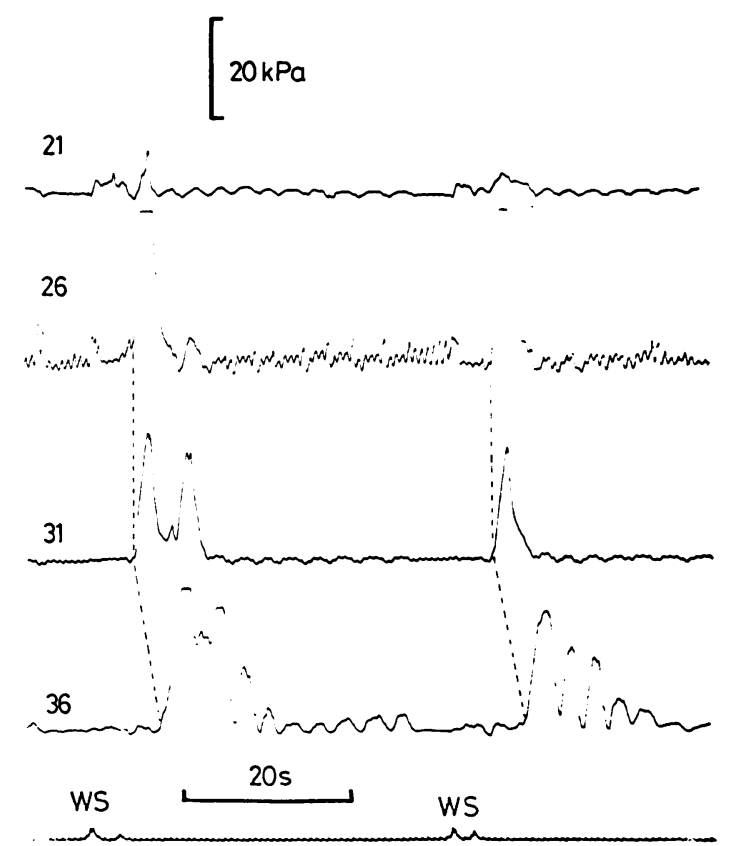

Fig. 1 'Interrupted peristalsis' with 'slow distal propagation'. Tracing, from patient with IDOS, shows, from above downwards, respiration (inspiration downwards), oesophageal pressures $21,26,31$ and $36 \mathrm{~cm}$ from incisors, and neck myograph. 'WS' represents 'wet' swallow ( $5 \mathrm{ml}$ of water). The interrupted lines connect the points of onset of rapid pressure rise in the three lowest pressure tracings. After each swallow, pressure rises simultaneously at 26 and $31 \mathrm{~cm}$. The onset of pressure rise at $36 \mathrm{~cm}$ is delayed, with time intervals between 31 and $36 \mathrm{~cm}$ being $3 \cdot 2$ and 3.6 seconds after first and second swallows respectively. Contractions at $36 \mathrm{~cm}$ are truly repetitive (cf. Fig. 4). 
which are almost invariably less than mid-respiratory and end-inspiratory pressures. LOS relaxation in response to swallowing occurred in all patients, but was judged to be incomplete in eight.

'Interrupted peristalsis'-defined as simultaneous rise in pressure at two or more proximal recording points with a later pressure rise at a distal recording point-was observed in seven of the 12 patients. In each it was an intermittent phenomenon. It is illustrated in Fig. 1. 'Slow distal propagation', also shown in Fig. 1, and defined as a time interval between onset of pressure rise at the two most distal recording points which exceeded the greatest value $(2.4 \mathrm{~s})$ recorded in any of the normal subjects, was observed in seven patients with IDOS. The maximum interval in these seven patients ranged between 3.4 and 6.4 seconds. Again, 'slow distal propagation', like 'interrupted peristalsis', was intermittent. It is important to note that six of the seven patients with 'slow distal propagation' also had 'interrupted peristalsis'.

Maximum values for distal oesophageal contractile amplitude, duration, and propagation time are shown in Fig. 2. Values for amplitude and duration exceeded, often by a substantial margin, the highest normal values in nine and 11 patients with IDOS respectively.

\section{Discussion}

The normal subjects referred to in this study tended to be younger than, and were not sex-matched with,

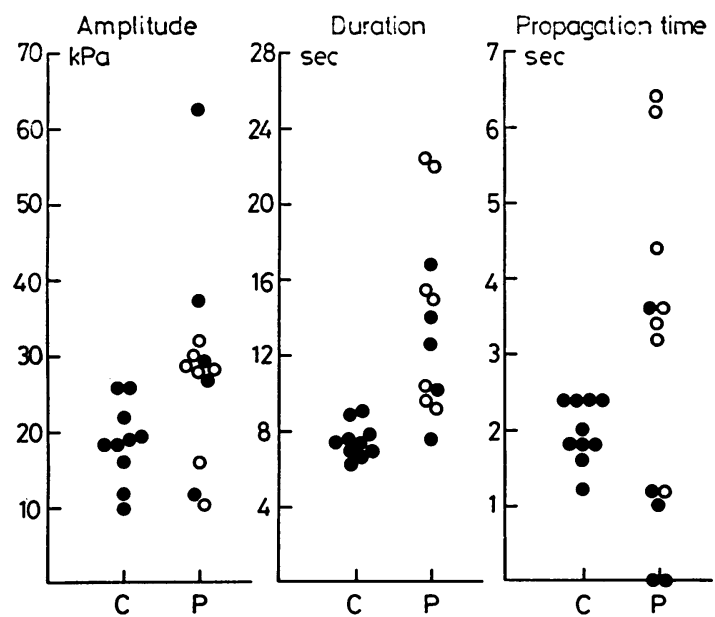

Fig. 2 Maximum values for amplitude, duration, and propagation time of distal oesophageal contractions in controls (C) and patients with IDOS (P). Patients with 'interrupted peristalsis' are represented by the open circles.

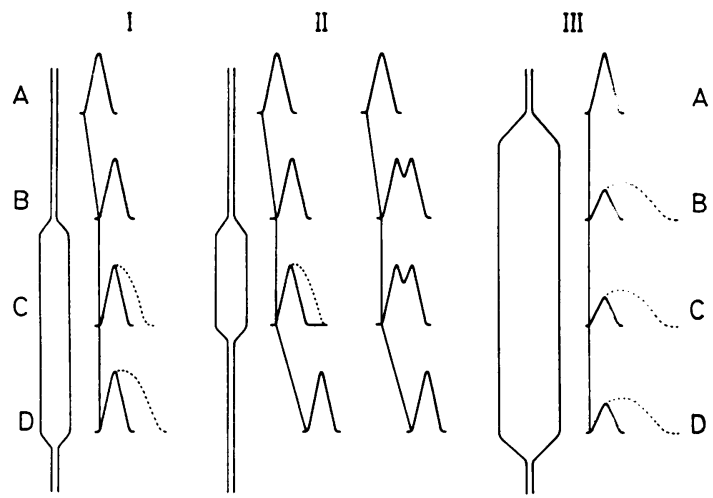

Fig. 3 An explanation for 'simultaneous contraction'. $I$, to left of Figure, represents typical diffuse oesophageal spasm. II, in centre of Figure, represents spasm with 'interrupted peristalsis' and 'slow distal propagation'. III, to right of Figure, represents achalasia. See text for detailed explanation.

the patients with IDOS. For this reason, it could be argued that they do not represent an ideal control group. However, results reported recently by Weihrauch et al. $^{7}$ would suggest that age and sex per se have little effect on contractile function of the oesophagus; and the only age-related change noted by Hollis and Castell $^{8}$ was a reduction in the amplitude of oesophageal contraction. Data from healthy individuals are included in this report primarily to provide a general frame of reference as an aid to the interpretation of measurements obtained in the patients with IDOS. This is important because normal values obtained in any one laboratory depend to a significant degree upon the methods and equipment used. ${ }^{9}$

The primary purpose of this paper is to draw attention to two manometric abnormalities-'interrupted peristalsis' and 'slow distal propagation'which were observed, almost always in association, in approximately half of a group of 12 patients with IDOS. At first sight, one might interpret the manometric phenomenon of 'interrupted peristalsis' to represent a contraction which passes sequentially along the proximal oesophagus, then occurs simultaneously in a segment of oesophagus, and then again becomes sequential at a lower level. Although our understanding of oesophageal peristalsis is limited, this sequence of events seems inherently improbable. An alternative explanation is presented in Fig. 3. The central concept is that 'aperistalsis' - that is to say, lack of axially sequential contraction-is apparent rather than real. It is proposed that the recording, during manometry, of a simultaneous rise in pressure at two or more points along the length of the oesophagus is due not to simultaneous onset 
of contraction at those points but to the transmission of pressure generated at a proximal point to more distal points. This hypothesis requires the existence, either transient at the time of contraction, or persistent, of a cavity below the level of the proximal pressure sensor and containing within it the distal pressure sensor(s). In the first illustration (I) of Fig. 3 , a contraction progresses normally from $\mathrm{A}$ to $\mathrm{B}$. The oesophageal lumen below point $B$ is open, so pressure rises simultaneously at $\mathrm{B}, \mathrm{C}$, and $\mathrm{D}$. If true contraction at $C$ and $D$ is absent, the configuration of the pressure waves at $\mathrm{B}, \mathrm{C}$, and $\mathrm{D}$ will be similar. However, if significant contraction occurs at $\mathrm{C}$ and

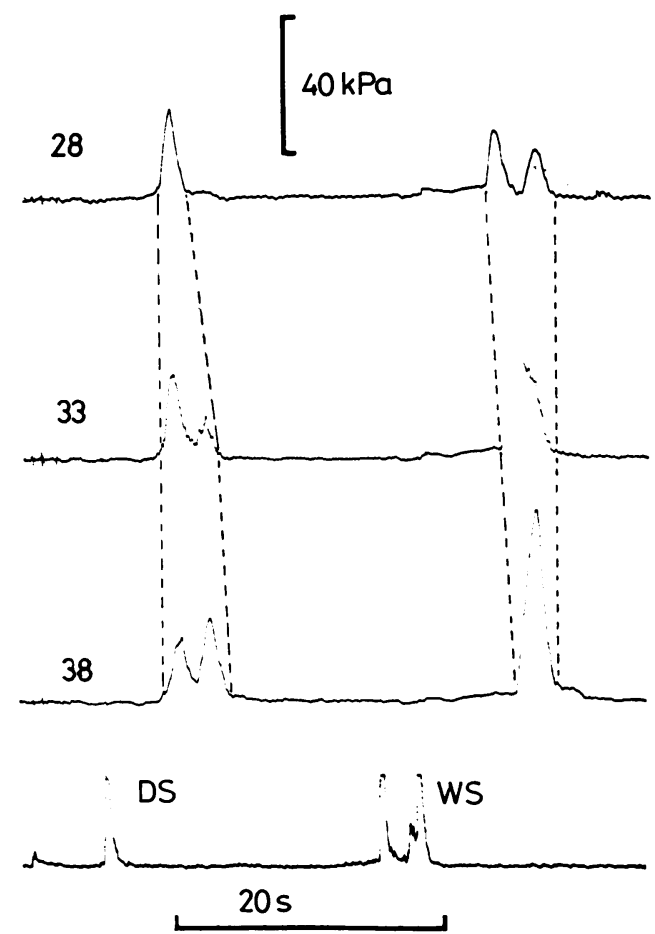

Fig. 4 The double-peaked pressure wave. Tracing from patient with IDOS. The interrupted lines join the beginning and end of pressure waves at the three recording points. After the 'dry' swallow (DS) pressure rises simultaneously at all three points. The first peak at 33 and $38 \mathrm{~cm}$ is due to pressure transmitted from $28 \mathrm{~cm}$. The second peaks at 33 and $38 \mathrm{~cm}$ are sequential, and are due to independent contraction at those points. After the 'wet' swallow (WS), pressure rises sequentially at all three points, and is due to progressive contraction.

Pressure generated by the unusually powerful contraction at $38 \mathrm{~cm}$ is transmitted upwards. It distorts the descending limb of the pressure wave at $33 \mathrm{~cm}$, and causes a distinct second peak at $28 \mathrm{~cm}$. In this case, the pressure wave ends simultaneously at the three points. These are not true repetitive contractions.
$\mathrm{D}$, onset of pressure rise at $\mathrm{B}, \mathrm{C}$, and $\mathrm{D}$, will remain simultaneous, but the duration of the pressure wave will increase progressively from B to D (hatched lines). Thus, the pressure wave recorded at any given point may be a composite, representing the summation of pressures generated not only at that point itself, but also above it, below it, or both. In section II of Fig. 3, sequential pressure rises are recorded at points A and B. A cavity exists below B, so that pressure rises simultaneously at points $B$ and $C$. Pressure generated at $B$ is not transmitted to D, as the oesophageal lumen in closed between $C$ and $D$. However, if true contraction occurs at $D$, the onset of pressure rise at that point will be not only sequential, but will appear delayed relative to the pressure wave at point $C$, onset of which reflects contraction at puint $\mathrm{B}$. This hypothesis therefore explains not only 'interrupted peristalsis' but also 'slow distal propagation'; and, if correct, it implies that peristalsis is not in fact 'interrupted' and propagation of contraction in the distal segment is not necessarily slow. The right half of section II of Fig .3 depicts another phenomenon that may be observed if points $B$ and $C$ are in open luminal continuity. Pressure generated by contraction at point $B$ causes a simultaneous rise in pressure at C. Subsequent contraction at $\mathrm{C}$ will, in turn, if the oesophageal lumen remains open, cause a simultaneous rise in pressure at $B$. Thus, the pressure wave at each point will have two peaks. An example of this rather commonly observed phenomenon is shown in Fig. 4. Section III of Fig. 3 depicts the situation in achalasia. If the entire ossophageal lumen is dilated and remains open, the pressure wave generated by contraction at the most proximal recording point (A) will be transmitted to $B, C$, and $D$. Thus, the entire oesophageal body will appear to contract simultaneously. If oesophageal muscle below point $A$ retains the ability to contract, the pressure wave at distal recording points will appear abnormally long (hatched lines). Contractions will appear feeble, as pressure is dissipated in the large volume of the dilated oesophagus. This general hypothesis is supported by recent observations $\mathbf{~}^{1011}$ that peristalsis may 'return' after successful treatment of achalasia. Such treatment depends upon relief of distal obstruction (at the LOS) and is accompanied by a reduction of oesophageal calibre. The basic conclusion of this hypothesis is that 'aperistalsis' may be a figment of the manometrist's imagination. The corollaries are that a simultaneous rise in pressure at two or more levels does not necessarily mean simultaneous contraction; that a pressure wave with two or more peaks does not necessarily indicate that the oesophagus at a given level has contracted more than once; and that an abnormally long pressure wave at 
a given level does not necessarily imply that the duration of muscular contraction at that same level is abnormal. I would emphasise, however, that the duration of contıaction is truly prolonged in at least some patients with IDOS; and that true repetitive contractions undoubtedly do occur, not only in the lower oesophagus in certain patients with IDOS, but also in the proximal oesophagus in some patients with scleroderma. Such contractions probably represent a response to incomplete oesophageal emptying.

The relationship between achalasia, IDOS, and other ress well-classified idiopathic disorders of oesophageal motility remains controversial. Arguing from the assumptions discussed above, I would propose that these disorders may share a common pathogenesis. The basic postulates are that the initial abnormality in all these disorders may be functional obstruction at the level of the LOS; and that abnormalities of motility in the oesophageal body may be secondary consequences of this obstruction. Patients with achalasia have, by definition, failure of LOS relaxation which, as determined by manometry, may be partial or complete. Such dysfunction of the LOS is usually present in "vigorous achalasia' ${ }^{12}$ and frequently present in IDOS. ${ }^{132}$ The finding of 'normal' LOS relaxation in some patients with IDOS means only that the LOS has relaxed to the point where its luminal diameter is as great as that of the recording cathei: Hence, rormal relaxation on manometry should not necessar $1 . y$ be equated with normal opening of the LOS. As smooth muscle undergoes hypertrophy in response to obstruction, it is germane to note that oesophageal muscular hypertrophy occurs in both achalasia ${ }^{1415}$ and IDOS. ${ }^{16-19}$ If the hypothesis is correct, the likelihood that a patient will present with, or progress to, florid achalasia will depend upon the magnitude of resting LOS pressure, the degree and duration of LOS dysfunction, and the stamina of the oesophageal body. The following sequence can be envisaged. The initial response to functional obstruction at the LOS will be hypertrophy of the oesophageal muscle. So long as the capacity for luminal obliteration during contraction is retained, manometry will show forceful but sequential contractions, and the oesophagus, may be characterised as a "high squeezer'4 or "nutcracker'. ${ }^{5}$ As this capacity is lost in the lower oesophagus, manometry will reveal simultaneous pressure waves distally, and may also show 'interrupted peristalsis' and 'slow distal propagation'. As loss of this capacity for luminal obliteration extends upwards, manometric abnormalities will evolve through the spectrum of typical diffuse oesophageal spasm, vigorous achalasia, and achalasia. Progression through this spectrum may be halted at any stage if the oesophageal body has adequate reserve, or if the degree of LOS dysfunction decreases. In this connection, it may be pertinent that sliding hiatal hernia is very rarely associated with achala$\mathrm{sia}^{20}$ but is common in IDOS. If one believes, as I do, that the strength of the LOS, and its resting pressure as recorded by infused-catheter manometry, depend not only on the intrinsic sphincter, but also on the external structures, such as diaphragmatic crura, which normally buttress it, then the development of a hernia in a patient with IDOS may reduce the degree of functional LOS obstruction and help to prevent progression to achalasia.

Patients with symptomatic IDOS often do not respond well to medical therapy. In those whose symptoms are severe and refractory, a long myotomy may be performed. The results of this procedure are frequently unsatisfactory, with benefit occurring in approximately two-thirds of patients subjected to it. ${ }^{21}$ If the hypothesis outlined above is correct, it would be more logical to perform a short myotomy of the type used in achalasia, as extension of the myotomy above the LOS would be expected only to reduce oesophageal propulsive force.

\section{References}

${ }^{1}$ Creamer B Donoghue FE, Code CF. Pattern of esophageal motility in diffuse spasm. Gastroenterology 1958, 34: 782-96.

${ }^{2}$ DiMarino AJ, Cohen S. Characteristics of lower esophageal sphincter function in symptomatic diffuse esophageal spasm. Gastroenterology 1974; 66: 1-6.

${ }^{3}$ Mellow MH. Symptomatic diffuse esophageal spasm. Manometric follow-up and response to cholinergic stimulation and cholinesterase inhibition. Gastroenterology 1977; 73: 237-40.

${ }^{4}$ Pope CE II. Abnormalities of peristaltic amplitude and force-a clue to the etiology of chest pain? In: Vantrappen G, ed. Proceedings of the Fifth International Symposium on Gastrointestinal Motility. Herentals, Belgium: Typoff Press, 1975: 380-6.

${ }^{5}$ Benjamin SB, Gerhardt DC, Castell DO. High amplitude peristaltic esophageal contractions associated with chest pain and/or dysphagia. Gastroenterology 1979; 77: 478-83.

${ }^{6}$ Kaye MD, Hoehn MM. Esophageal motor dysfunction in Parkinson's disease. In: Vantrappen G, ed. Proceedings of the Fifth International Symposium on Gastrointestinal Motility. Herentals, Belgium: Typoff Press, 1975: 393-9.

${ }^{7}$ Weihrauch TR, Vallerins P, Alpers H, Ewe K. Assessment of various factors influencing esophageal pressure measurement. II. Significance of physiological factors in intraluminal manometry. Klin Wochenschr 1980; 58: 287-92.

${ }^{8}$ Hollis J, Castell DO. Esophageal function in elderly men: a new look at presbyesophagus. Ann Intern Med 1974; 80: 371-4. 
${ }^{9}$ Dodds WJ. Instrumentation and methods for intraluminal esophageal manometry. Arch Intern Med 1976; 136: 515-23.

${ }^{10}$ Mellow MH. Return of esophageal peristalsis in idiopathic achalasia. Gastroenterology 1976; 70: 1148-51.

"Vantrappen G, Janssens J, Hellemans J, Coremans G. Achalasia, diffuse esophageal spasm, and related motility disorders. Gastroenterology 1979; 77: 450-7.

${ }^{12}$ Sanderson DR, Ellis FH, Schlegel JF, Olsen AM. Syndrome of vigorous achalasia: clinical and physiologic observations. Dis Chest 1967; 52: 508-17.

${ }^{13}$ Kaye MD. Dysfunction of the lower esophageal sphincter in disorders other than achalasia. Am J Dig Dis 1973; 18: 734-45.

${ }^{14}$ Shattock SG. Remarks on dilatation of the oesophagus. Proc Roy Soc Med (Section on Laryngology) 1919; 12: 95-100.
${ }^{15}$ Rake GW. On the pathology of achalasia of the cardia. Guy's Hosp Rep 1927; 77: 141-50.

${ }^{16}$ Johnston AS. Diffuse spasm and diffuse muscular hypertrophy of the lower esophagus. Br J Radiol 1960; 33: 723-35.

${ }^{17}$ Craddock DR, Logan A, Walbaum PR. Diffuse oesophageal spasm. Thorax 1966; 21 : 511-7.

${ }^{18}$ Ramsay BH. Esophageal hypertrophy with esophagospasm (curling), Ann Thorac Surg 1967; 4: 66-70.

${ }^{19}$ Gillies M, Nicks R, Skyring A. Clinical, manometric and pathological studies in diffuse oesophageal spasm. Br Med J 1967; 2: 527-30.

${ }^{20}$ Binder HJ, Clement AR, Thayer WR, Spiro HM. Rarity of hiatus hernia in achalasia. $N$ Engl J Med 1965; 272: 680-2.

${ }^{21}$ Ellis FH Jr, Olsen AM, Schlegel JF, Code CF. Surgical treatment of esophageal hypermotility disturbances. JAMA 1964; 188: 862-6. 